\title{
To Reform Pharmacy Reimbursement Begin with the Basics
}

\author{
Michael T. Rupp, PhD, FAPhA
}

Rupp's Viewpoint

$\mathrm{I}$ am encouraged by the perspectives of Drs. Nightengale and Trygstad in the preceding viewpoints regarding the prospects of emerging professional services to produce new revenue streams for community pharmacies. However, I am disappointed that neither author addresses the need to reform payment for conventional services related to dispensing in third-party benefit programs. Indeed, there seems to be an implied concession that equitable compensation for this bedrock of community pharmacy revenue has been irrevocably lost. For the sake of community pharmacies and the health and safety of their patients, I hope that is not the case.

Some historical perspective is helpful to understand the current state of pharmacy reimbursement for dispensing-related services in third-party benefit programs.

\section{How Managed Care Changed Pharmacy Reimbursement}

Before managed care, pharmacies and other health care providers billed using "reasonable and customary" (R\&C) charges. These R\&C charges included the acquisition cost of the drug product and a markup on the product to cover the operating costs incurred to dispense the medication to patients in compliance with all applicable laws, regulations, and practice standards. Because every pharmacy's drug acquisition and operating costs are different, both components of the R\&C charge were necessarily different for each. Finally, the R\&C charge included what the pharmacy owner/manager considered to be a reasonable profit margin for the transaction.

With the introduction of managed care, pharmacy benefits managers concluded that R\&C charges, while customary, were not reasonable. This led to the introduction of the standard 2-component prescription reimbursement model that continues to be used in pharmacy benefit contracts today.

The first component is typically expressed as a discount off a published index price for the dispensed medication, such as average wholesale price (AWP) or wholesale acquisition

\section{J Manag Care Spec Pharm. 2020;26(6):713-15}

Copyright $\odot 2020$, Academy of Managed Care Pharmacy. All rights reserved. cost (WAC). This component of the formula is intended to reflect an estimate of the pharmacy's actual acquisition cost. Naturally, it cannot accurately reflect every pharmacy's acquisition cost, since each is different.

The second component of the reimbursement formula is typically a flat dispensing fee. The dispensing fee was originally intended to reflect the operating costs incurred by the pharmacy to dispense the medication. But once again, since each pharmacy's operating costs are different, a flat fee cannot reasonably be expected to do so.

\section{The Turning Point}

While this reimbursement model was clearly inequitable to pharmacies with higher product acquisition or operating costs, in the early days of managed pharmacy care the net compensation was generally acceptable to pharmacy owners because they were routinely purchasing product for a greater discount off AWP or WAC than the formula was paying.

That is, pharmacies' profit was effectively hidden in the ingredient component of the reimbursement formula because their true acquisition costs were significantly less. This is important because very early in the evolution of managed pharmacy benefit plans, the average cost incurred by pharmacies to dispense a prescription quickly began to exceed the flat dispensing fee that pharmacies were receiving. As a result, pharmacies became increasingly dependent on the "spread" between true acquisition cost and the payment provided in the ingredient component of the formula.

The critical turning point came when third-party prescription benefit contracts began to demand ever-greater discounts on the ingredient component, thereby progressively eliminating the sole source of pharmacy profitability. Increasingly, the ingredient component of pharmacy reimbursement came to be very near, and sometimes even below, the actual acquisition cost. Since the flat dispensing fee component had always been inadequate to cover the true costs of dispensing, these AWP $-\mathrm{X} \%+\$ \mathrm{Y}$ payment formulas became cynically known as "AWP - a lot + a little" and began to seriously threaten the financial viability of many community pharmacies, especially independents. 
I can clearly recall a widely held opinion some years ago that if gross margins ever fell below 30\% it would be the death knell for community pharmacy. That did not happen, as the average gross margin among independent pharmacies is now reported to be below $22 \% .{ }^{1}$ Instead, community pharmacies responded by driving down operating costs-especially including personnel-while ramping up prescription volume. But, while this strategy provided temporary relief, it is not perpetually sustainable nor is it in the best interests of practicing pharmacists or their patients.

\section{The Importance of Getting the Basics Right}

Among the immutable truths I have learned during my 40 plus years in pharmacy are the following: First, you cannot legislate or regulate your way to good pharmacy practice. You can incentivize and reward it, but you cannot successfully mandate it. Second, good pharmacy practice takes more time than poor pharmacy practice. Third, for the time needed to provide high quality care to be consistently available in the real world of retail pharmacy, it must be compensated. Otherwise, this time will simply not be consistently available to pharmacists and to believe otherwise is an exercise in self-delusion.

It is dangerously naive to believe that quality pharmacy care will consistently occur in an environment where production volume of an undifferentiated commodity is the only valued metric. Unfortunately, that is perhaps the most pernicious effect that managed care has had on routine prescription pharmaceutical care in the community setting.

I absolutely agree that we should pursue new professional opportunities and related revenue streams for community pharmacy. However, real harm results from ignoring the need to ensure that pharmacies consistently get the basics right. As someone who has done a significant amount of expert witness work on medication error lawsuits, I can tell you that it is the routine failure of basic responsibilities such as accurate dispensing, thorough prospective drug utilization review (proDUR), and complete and consistent patient counseling that are responsible for the vast majority of medication errors and their adverse consequences on patients.

\section{Toward a New Payment Model}

So, what is to be done? I believe there are 2 areas in need of reform. First, we need accurate metrics with which to measure the quality of medication dispensing, pro-DUR, and patient counseling provided by community pharmacies. ${ }^{2,3}$ In a recent commentary, the director of Measurement Outcomes Research for the Pharmacy Quality Alliance recommended that "a multistakeholder, consensus-based, transparent approach should be used to develop pharmacist/pharmacy quality measures." ${ }^{\text {"M }}$ My response: Great idea, so why has this obvious need still not been addressed? ${ }^{5}$
With a set of reliable quality metrics with which to distinguish pharmacies on core practice competencies, we then have the foundation for a new prescription-dispensing reimbursement model that would differentially compensate pharmacies for the differential value they create in the delivery of conventional prescription pharmaceutical care.

Our second area of reform, therefore, is to create a new payment model that uses these quality metrics to equitably compensate pharmacies that are able to consistently meet higher performance standards. Specifically, I propose a 3-component formula.

First, the ingredient component of reimbursement must reflect verifiable actual acquisition cost of the drug product plus a mutually acceptable gross margin. Second, there must be a dispensing fee that reflects the operating costs incurred by pharmacies to dispense a prescription, including the provision of standard pro-DUR activities and routine patient counseling. This fee would initially be based on a cost-of-dispensing analysis among participating pharmacies, then annually adjusted using a general index of health care inflation.

These 2 components represent the standard reimbursement formula and provide adequate compensation for routine prescription care. However, during the course of a typical practice day, there are exceptional events that require additional pharmacist time and therefore merit additional payment. As a third component, a supplementary professional fee should be available to recognize the cost and value of pharmacists' time when they must stop the efficient machinery of the dispensing process to resolve medication-related problems identified during pro-DUR or perform above normal counseling for at-risk patients. These targeted professional interventions would be documented and billed using features currently available in the telecommunications standard from the National Council for Prescription Drug Programs that all community pharmacies use to submit third-party claims for conventional prescription care.

While I am disappointed that it has still not occurred, I believe a true performance-based network of community pharmacies will eventually be created that maintains participation requirements for all members using quality metrics such as those previously described. Such a network should be capable of demonstrating improvements in desired clinical, economic, and/or humanistic outcomes and significant reductions in undesired outcomes when compared with conventional community pharmacy care.

A true performance-based network would fundamentally alter the competitive playing field in community pharmacy by introducing a criterion other than cost-that is, quality. When that happens, everything changes-payers who desire an undifferentiated commodity product at the lowest possible cost can still follow their utilities, but those who recognize the value of consistently higher quality care to ensure optimal medication use can also follow theirs. 
Ultimately, the market will decide, and that is as it should be. But for a market to make a rational choice it must be given options, and a true performance-based network of community pharmacies that are consistently getting the basics right is the first step.

\section{Author}

MICHAEL T. RUPP, PhD, FAPhA, Midwestern University College of Pharmacy, Glendale, Arizona.

AUTHOR CORRESPONDENCE: Michael T. Rupp, BSPharm, PhD, FAPhA, Midwestern University College of Pharmacy,

19555 N. 59th Ave., Glendale, AZ 85383. Tel.: 623.572.3528;

E-mail: MTRUPP@midwestern.edu.

\section{DISCLOSURES}

No funding supported the writing of this commentary. The author has nothing to disclose.

\section{REFERENCES}

1. National Community Pharmacy Association. 2019 NCPA Digest. Available at: http://www.ncpa.co/pdf/digest/2019/2019-digest.pdf. Accessed April 27, 2020.

2. Rupp MT. Assessing the quality of care in pharmacy: remembering Donabedian. J Manag Care Spec Pharm. 2018;24(4):354-56. Available at: https://www.jmcp.org/doi/10.18553/jmcp.2018.24.4.354.

3. Rupp MT. 10 ways to improve medication safety in community pharmacies. J Am Pharm Assoc (2003). 2019;59(4):474-78.

4. Campbell PJ. Adherence: an important metric to assess pharmacist value in quality care. J Manag Care Spec Pharm. 2019(10);25:1044-45. Available at: https://www.jmcp.org/doi/10.18553/jmcp.2019.25.10.1044.

5. Rupp MT. Assessing the quality of pharmacy care: it's not about adherence. J Manag Care Spec Pharm. 2019;25(10):1046-47. Available at: https:// www.jmcp.org/doi/10.18553/jmcp.2019.25.10.1046. 